\title{
Optimal numbers of mature oocytes to produce at least one or multiple top-quality day-3 embryos in normal responders
}

\author{
Yoo Jin Shim ${ }^{1,2}$, Yeon Hee Hong ${ }^{1,2}$, Seul Ki Kim ${ }^{1,2}$, Byung Chul Jee ${ }^{1,2}$ \\ 'Department of Obstetrics and Gynecology, Seoul National University Bundang Hospital, Seongnam; '2Department of Obstetrics and Gynecology, \\ Seoul National University College of Medicine, Seoul, Korea
}

Objective: We attempted to identify the optimal cutoff numbers of mature oocytes that would produce at least one or multiple top-quality (grade A) day-3 embryos in normal responders undergoing stimulated in vitro fertilization (IVF) cycles.

Methods: We selected 210 fresh IVF cycles performed in 170 infertile women at a single center from January 2014 to November 2019. Four to 14 (total) oocytes were obtained in all cycles after conventional ovarian stimulation. A receiver operating characteristic curve analysis was performed to find the moderate and extreme cutoff numbers of mature oocytes that would produce $\geq 1, \geq 2, \geq 3, \geq 4$, and $\geq 5$ top-quality embryos.

Results: The cutoff number of mature oocytes was significantly correlated with the number of top-quality embryos $(r=0.467, p=0.000)$. The moderate cutoff number of mature oocytes was $\geq 3, \geq 5, \geq 5, \geq 6$, and $\geq 6$ for obtaining $\geq 1, \geq 2, \geq 3, \geq 4$, and $\geq 5$ top-quality embryos, respectively. The extreme cutoff number of mature oocytes was $\geq 9, \geq 9, \geq 10, \geq 10$, and $\geq 11$ for obtaining $\geq 1, \geq 2, \geq 3$, $\geq 4$, and $\geq 5$ top-quality embryos, respectively.

Conclusion: We present the optimal cutoff numbers of mature oocytes that would yield $\geq 1, \geq 2, \geq 3, \geq 4$, and $\geq 5$ top-quality embryos with 95\% specificity. Our findings could help infertility clinicians to set target mature oocyte numbers in women undergoing stimulated IVF cycles.

Keywords: Embryo; In vitro fertilization; Infertility; Oocyte

\section{Introduction}

In in vitro fertilization (IVF) cycles, the pregnancy rate depends on the number of embryos transferred [1-3]. More specifically, the pregnancy rate after IVF depends on the number of good-quality embryos transferred [4-7]. Volpes et al. [8] reported that the number of good-quality embryos available on day 3 was a strong predictor of

Received: October 13, 2019· Revised: February 10, 2020 · Accepted: February 20, 2020 Corresponding author: Byung Chul Jee

Department of Obstetrics and Gynecology, Seoul National University Bundang Hospital, 82 Gumi-ro 173beon-gil, Bundang-gu, Seongnam 13620, Korea Tel:+82-31-787-7254 Fax:+82-31-787-4054 E-mail: blasto@snubh.org

This is an Open Access article distributed under the terms of the Creative Commons Attribution Non-Commercial License (http://creativecommons.org/licenses/by-nc/4.0/) which permits unrestricted non-commercial use, distribution, and reproduction in any medium, provided the original work is properly cited. both the pregnancy rate and the implantation rate. In their study, when more than three good-quality embryos were available, the overall pregnancy rate was $64.3 \%$, whereas it was only $39.5 \%$ when three good-quality embryos were available [8]. In a meta-analysis, the total number of good-quality embryos was found to be the most important predictor of the clinical pregnancy rate after IVF or intracytoplasmic sperm injection (ICSI) among 27 candidate variables [9]. Terriou et al. [7] demonstrated that the mean score of transferred embryos was a better predictor of pregnancy than the number of transferred embryos itself and/or the age of the woman undergoing IVF. Lee et al. [10] also suggested that the score of the three best embryos on day 3 was more closely correlated with the pregnancy rate after IVF than the number of good-quality embryos or the total embryo score. 
Recently, the oocyte number has been reported to be closely related with the pregnancy rate after IVF. Sunkara et al. [11] found that the best chance of live birth was seen with approximately 15 oocytes. Magnusson et al. [12] reported that the live birth rate increased up to 11 oocytes and plateaued after that number. Steward et al. [13] showed that the highest pregnancy rate (30.8\%) was found when between 11 and 15 mature oocytes were available for ICSI. Considering the above reports, the primary goal in stimulated IVF cycles is to obtain one or multiple good-quality embryos, and for that goal, the retrieval of multiple oocytes may be an important prerequisite. While a high number of oocytes clearly is a strong predictor of multiple good-quality embryos; however, the specific number of oocytes needed to obtain one or multiple good-quality embryos has not been established.

In this study, we attempted to identify the specific cutoff numbers of mature oocytes that would be appropriate for obtaining at least 1 , $2,3,4$, or 5 top-quality embryos in normal responders undergoing stimulated IVF cycles.

\section{Methods}

\section{Study subjects}

We retrospectively selected 210 stimulated IVF/ICSI cycles from January 2014 to November 2019 among patients treated at Seoul National University Bundang Hospital. Cycles in which 4 to 14 total oocytes were retrieved after conventional ovarian stimulation were included in the analysis. The indications for IVF/ICSI were unexplained infertility ( 52 cycles), tubal factor infertility (39 cycles), decreased ovarian response (38 cycles), male factor infertility ( $23 \mathrm{cy}-$ cles), endometriosis (11 cycles), ovulation factor infertility (9 cycles), uterine/endometrial factor infertility ( 9 cycles), and mixed causes (29 cycles). The Institutional Review Board of Seoul National University Bundang Hospital approved our study (IRB No. B 1910-571-107).

\section{Controlled ovarian hyperstimulation protocols}

A flexible gonadotropin-releasing hormone $(\mathrm{GnRH})$ antagonist protocol was used in all IVF/ICSI cycles. Gonadotropins were started on day 3 of the menstrual cycle and the doses were adjusted according to individual ovarian response. When the leading follicle reached a diameter of 14 mm, 0.25 mg/d of cetrorelix (Cetrotide; Merck Serono, Darmstadt, Germany) was started. When the leading follicle(s) reached a diameter of $18 \mathrm{~mm}, 250 \mu \mathrm{g}$ or $500 \mu \mathrm{g}$ of recombinant human chorionic gonadotropin (hCG; Ovidrel, Merck-Serono; 194 cycles), a GnRH agonist (Decapeptyl $0.2 \mathrm{mg}$, Ferring, Germany; 4 cycles) or $250 \mu \mathrm{g}$ of recombinant hCG+a GnRH agonist (Decapeptyl 0.2 mg; 12 cycles) was administered, and oocytes were retrieved $35-36$ hours later.

After collection of cumulus-oocyte complexes (COC), the maturity of oocytes was assessed according to the presence or absence of a germinal vesicle (GV) or the first polar body (PB) by stereomicroscopy. In most cases, oocyte maturity could be easily evaluated under stereomicroscopy on the basis of the cumulus pattern. In situations where the maturity was unclear due to a dark $\mathrm{COC}$ or blood clots, the oocytes were denuded using $85 \mathrm{IU} / \mathrm{mL}$ hyaluronidase (Cook, Bloomington, IN, USA) and mechanical pipetting. Immature oocytes were identified by the absence of the first PB and then classified as GVstage or metaphase I (MI)-stage depending on the presence of a visible GV. Culture of GV- and/or Ml-stage oocytes was carried out in maturation medium (Global Total; Life Global, Airdropped, Denmark) supplemented with recombinant follicle-stimulating hormone (75 $\mathrm{mlU} / \mathrm{mL}$, Merck-Serono) and recombinant hCG $(0.5 \mathrm{IU} / \mathrm{mL}$, Merck-Serono). The culture of all oocytes continued in $1 \mathrm{~mL}$ of maturation medium for up to 48 hours in an incubator (Mini Incubator; Cook Medical, Bloomington, IN, USA) with a triple-gas system $\left(6 \% \mathrm{CO}_{2}, 5 \% \mathrm{O}_{2}\right.$, $89 \% \mathrm{~N}_{2}$ with high humidity).

Oocytes were then fertilized by the conventional method (77 cycles), ICSI (115 cycles), or half ICSI (18 cycles). The indications for ICSI were as previously described: (1) severe male factor infertility; (2) a $\leq 20 \%$ fertilization rate in a prior conventional insemination cycle; (3) repeated implantation failure ( $\geq 3$ times); (4) advanced maternal age ( $\geq 40$ years); (5) presence of endometrioma on the day of oocyte retrieval; or (6) poor-quality oocytes that included blood clots attached to the cumulus oophorus at the time of retrieval [14]. Normal fertilization was confirmed when two distinct pronuclei and a second PB were present. The fertilized oocytes were cultured in the culture medium (Global Total; LifeGlobal, Guilford, CT, USA) in an incubator with a triple-gas system.

\section{Embryo quality assessment}

Embryo quality was assessed at day 3 after insemination using the following morphological grading system: grade $\mathrm{A}$, equal-sized blastomeres without fragments or apparent morphological abnormalities; grade B, equal-sized blastomeres and $<20 \%$ of fragments without apparent morphological abnormalities; grade $C$, irregularity of blastomeres and $20 \%$ to $50 \%$ of fragments without apparent morphological abnormalities; grade $\mathrm{D}$, irregularity of blastomeres and $>50 \%$ fragments with apparent morphological abnormalities [15]. In the present study, grade A embryos were regarded as top-quality embryos.

\section{Data analysis}

Statistical analysis was performed using IBM SPSS ver. 25.0 (IBM Corp., Armonk, NY, USA). Pearson correlation analysis was performed to elucidate which variables were associated with the number of topquality embryos. Receiver operating characteristic curve analysis was 
performed to determine the cutoff numbers of mature oocytes that would produce $\geq 1, \geq 2, \geq 3, \geq 4$, and $\geq 5$ top-quality embryos. The cutoff point using the Youden index was defined as the moderate cutoff. We also obtained the extreme cutoff values following the methodology that we used in a previous study [16]. To maximize the

Table 1. Demographic characteristics and cycle outcome parameters of all study subjects

\begin{tabular}{lc}
\hline Variable & Mean \pm SD (range) \\
\hline Female age (yr) & $36.2 \pm 4.7(21-46)$ \\
Male age (yr) & $38.9 \pm 4.8(24-58)$ \\
BMl of female partner $\left(\mathrm{kg} / \mathrm{m}^{2}\right)$ & $22.8 \pm 3.8(16.7-36.1)$ \\
Serum anti-Müllerian hormone level $(\mathrm{ng} / \mathrm{mL})$ & $3.1 \pm 2.5(0.12-12.4)$ \\
Sperm volume $(\mathrm{mL})$ & $2.9 \pm 1.5(0.5-7.5)$ \\
Sperm concentration (million/mL) & $104 \pm 109(2-1,024)$ \\
Sperm motility $(\%)$ & $45.5 \pm 18.8(0.3-93.2)$ \\
Total motile sperm count (million) & $157 \pm 242(0.02-2,666)$ \\
Total gonadotropin dosage (IU) & $1,997 \pm 711(300-4,050)$ \\
Number of total oocytes & $7.8 \pm 3.1(4-14)$ \\
Number of mature oocytes & $6.1 \pm 2.6(1-13)$ \\
Number of normally fertilized zygotes & $5.0 \pm 2.4(0-12)$ \\
Number of top-quality (grade A) embryos & $1.9 \pm 1.8(0-8)$ \\
Grade A embryo formation rate per normally & $37.5 \pm 28.9(0.0-100.0)$ \\
fertilized zygote (\%) & \\
\hline
\end{tabular}

SD, standard deviation; BMI, body mass index.

Table 2. Pearson correlation coefficients for the associations between the number of top-quality (grade A) embryos and other factors

\begin{tabular}{lcc}
\hline \multirow{2}{*}{ Variable } & \multicolumn{2}{c}{ Correlation coefficient } \\
\cline { 2 - 3 } & $r$ & $p$-value \\
\hline Female age & -0.025 & 0.719 \\
Body mass index of female partner & -0.066 & 0.344 \\
Serum anti-Müllerian hormone level & 0.094 & 0.180 \\
Total gonadotropin dosage & -0.033 & 0.633 \\
Number of mature oocytes & 0.467 & 0.000 \\
\hline
\end{tabular}

specificity (true negative rate), the extreme cutoff value was selected by establishing a false positive rate of $5 \%$ (i.e., a specificity of $95 \%$ ). A $p$-value $<0.05$ was considered to indicate statistical significance.

\section{Results}

The demographic characteristics and cycle outcome parameters of all study subjects are shown in Table 1. Pearson correlation coefficients are presented in Table 2. The number of mature oocytes showed a significant correlation with the number of top-quality embryos $(r=0.467, p=0.000)$. A regression line depicting the association between the number of mature oocytes and the number of top-

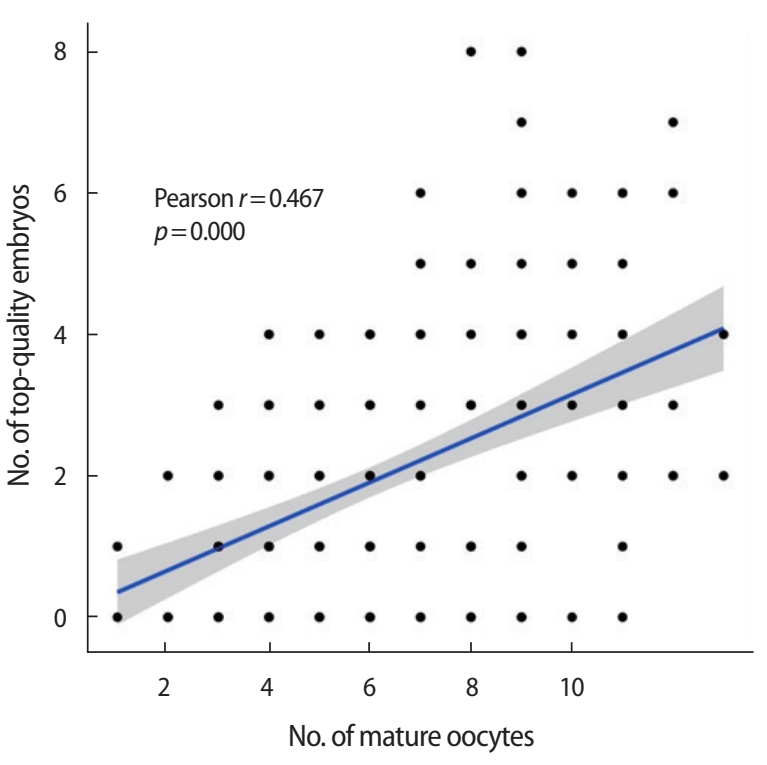

Figure 1. Matrix scatter plot and regression line showing the correlation between the number of mature oocytes and the number of topquality (grade A) embryos ( $r=0.467, p=0.000)$.

Table 3. Cutoff numbers of mature oocytes needed to obtain $\geq 1, \geq 2, \geq 3, \geq 4$, or $\geq 5$ top-quality (grade A) embryos

\begin{tabular}{llccccccc}
$\begin{array}{l}\text { Number of top- } \\
\text { quality (grade A) } \\
\text { embryos }\end{array}$ & $\begin{array}{c}\text { Cutoff of } \\
\text { mature oocytes }\end{array}$ & $\begin{array}{c}\text { Area under } \\
\text { the curve }\end{array}$ & $\begin{array}{c}95 \% \text { Confidence } \\
\text { interval }\end{array}$ & Sensitivity & Specificity & $\begin{array}{c}\text { Positive } \\
\text { predictive value }\end{array}$ & $\begin{array}{c}\text { Negative } \\
\text { predictive value }\end{array}$ & $p$-value \\
\hline$\geq 1$ & Moderate $\geq 3$ & 0.628 & $0.537-0.720$ & 88.3 & 29.8 & 57.6 & 18.8 & $<0.001$ \\
$\geq 1$ & Extreme $\geq 9$ & & & 12.9 & 95 & 86.4 & 24.7 & \\
$\geq 2$ & Moderate $\geq 5$ & 0.720 & $0.652-0.788$ & 67.0 & 65.4 & 34.0 & 33.6 & $<0.001$ \\
$\geq 2$ & Extreme $\geq 9$ & & & 26 & 95 & 79.5 & 57.2 & \\
$\geq 3$ & Moderate $\geq 5$ & 0.747 & $0.678-0.817$ & 78.5 & 61.4 & 13.6 & 52.3 & $<0.001$ \\
$\geq 3$ & Extreme $\geq 10$ & & & 17.6 & 95 & 60.9 & 72.7 & \\
$\geq 4$ & Moderate $\geq 6$ & 0.792 & $0.718-0.867$ & 77.8 & 70.7 & 6.1 & 64.6 & $<0.001$ \\
$\geq 4$ & Extreme $\geq 10$ & & & 25.0 & 95 & 47.8 & 86.6 & \\
$\geq 5$ & Moderate $\geq 6$ & 0.877 & $0.823-0.932$ & 100.0 & 68.2 & 0 & 77.2 & $<0.001$ \\
$\geq 5$ & Extreme $\geq 11$ & & & 27.8 & 95 & 33.3 & 93.3 & \\
\hline
\end{tabular}



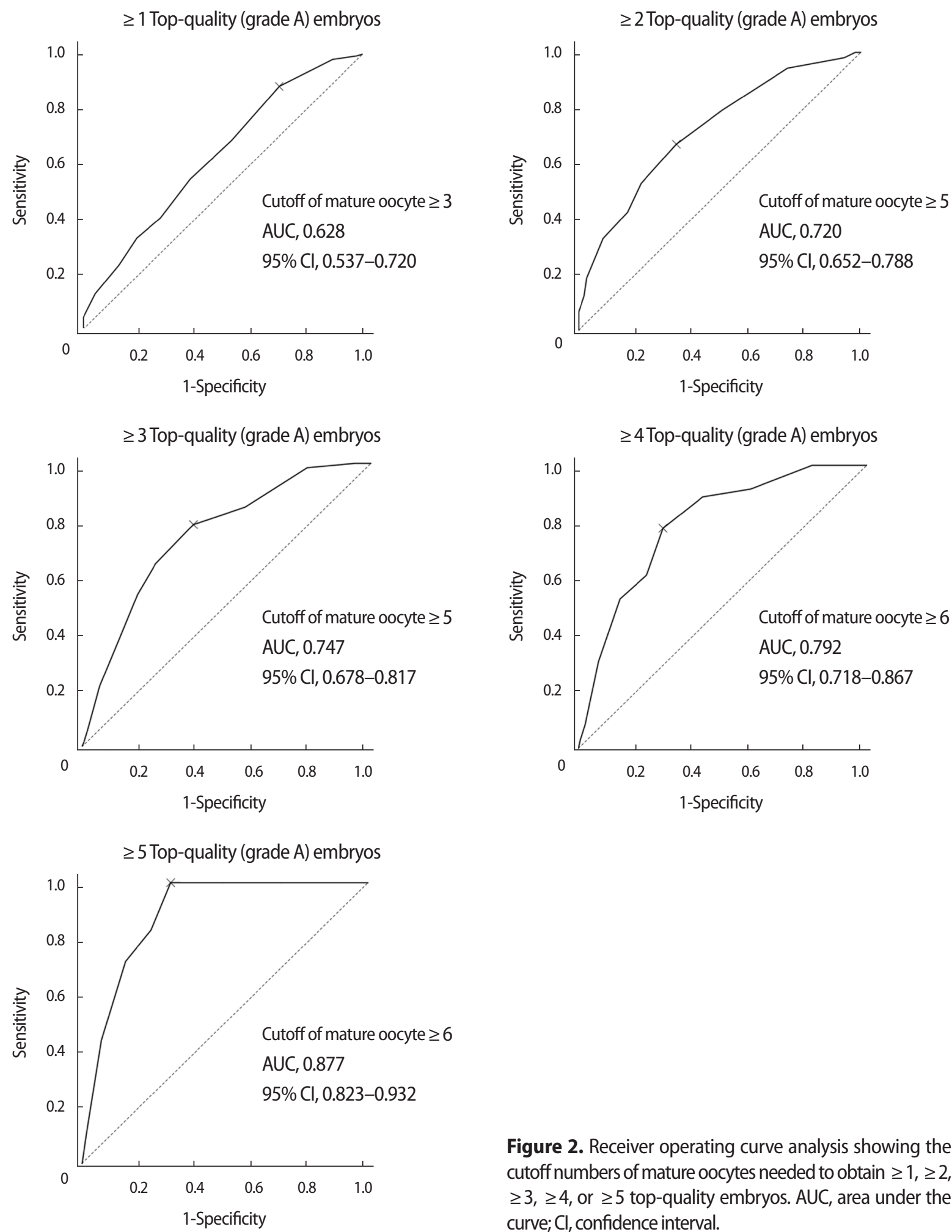

quality embryos is shown in Figure 1. At least one top-quality embryo was obtained in 163 cycles, at least two top-quality embryos were obtained in 106 cycles, at least three top-quality embryos were obtained in 65 cycles, at least four top-quality embryos were obtained in 36 cycles, and at least five top-quality embryos were ob-

Figure 2. Receiver operating curve analysis showing the cutoff numbers of mature oocytes needed to obtain $\geq 1, \geq 2$, $\geq 3, \geq 4$, or $\geq 5$ top-quality embryos. AUC, area under the curve; $\mathrm{Cl}$, confidence interval.

tained in 18 cycles.

As shown in Table 3, the moderate cutoff numbers of mature oocytes were $\geq 3, \geq 5, \geq 5, \geq 6$, and $\geq 6$ for obtaining $\geq 1, \geq 2, \geq 3, \geq 4$, and $\geq 5$ top-quality embryos, respectively. The extreme cutoff numbers of mature oocytes were $\geq 9, \geq 9, \geq 10, \geq 10$, and $\geq 11$ for ob- 
taining $\geq 1, \geq 2, \geq 3, \geq 4$, and $\geq 5$ top-quality embryos, respectively. Receiver operating curve analysis showing the cutoff numbers of mature oocytes needed to obtain $\geq 1, \geq 2, \geq 3, \geq 4$, or $\geq 5$ top-quality embryos are depicted in Figure 2 . When there were $\geq 9$ mature oocytes (44 cycles), the mean number of top-quality embryos was $3.3 \pm 2.2$, at least one top-quality embryo was obtained in $86.4 \%$ of the cycles, and at least two top-quality embryos were obtained in $79.5 \%$ of the cycles. When there were $\geq 10$ mature oocytes (23 cycles), the mean number of top-quality embryos was 3.5 \pm 2.1 , at least three top-quality embryos were obtained in $60.9 \%$ of the cycles, and at least four top-quality embryos were obtained in $47.8 \%$ of the cycles. When there were $\geq 11$ mature oocytes (15 cycles), the mean number of top-quality embryo was $3.5 \pm 2.2$, and at least five topquality embryos were obtained in $33.3 \%$ of the cycles.

\section{Discussion}

In the present study, we found that the presence of $\geq 9, \geq 10$, and $\geq 11$ mature oocytes strongly predicted the ability to produce $\geq 1-2$, $\geq 3-4$, and $\geq 5$ top-quality embryos, respectively, with a high probability. In most fresh IVF cycles, obtaining $\geq 1-2$ top-quality embryos may be ideal; thus, for this purpose, obtaining $\geq 9$ mature oocytes is recommended. When there were $\leq 8$ mature oocytes in the present study, the mean number of top-quality embryos was 1.6 1.4 (166 cycles). In contrast, when there were $\geq 9$ mature oocytes (44 cycles), the mean number of top-quality embryos was $3.3 \pm 2.2$.

Some investigators have reported that the live birth rate depends on the number of oocytes [11-13]. Retrieving more oocytes could result in more available good-quality embryos, which would lead to higher pregnancy and live birth rates. Cai et al. [17] suggested that the number of oocytes can be considered as a surrogate of embryo quality because an abundant number of oocytes predicts a larger pool of available embryos and a higher possibility of obtaining one or two good-quality embryos.

In the present study, the number of top-quality embryos was correlated with the number of mature oocytes. Female age, body mass index, serum anti-Müllerian hormone levels, and total gonadotropin dosage were not related with the number of top-quality embryos. Hsu et al. similarly reported that the number of total oocytes had a positive correlation with the number of good-quality embryos $(r=0.329, p<0.001)$ [18]. They also showed that the number of oocytes was positively correlated with pregnancy and implantation rates. A recent meta-analysis demonstrated that the number of total oocytes had strong positive associations with the presence of top- or good-quality day-2, day-3, day-5, and day-6 embryos [19]. In IVF cycles, it is important to obtain at least one good-quality embryo, but the quality of surplus embryos from the same oocyte retrieval cohort is also important. Surplus embryos could be cryopreserved and used later, thereby maximizing the overall pregnancy rate from the same oocyte retrieval cohort.

A limitation of our study is that we only included normal responders. If the study had included poor responders and/or high responders, the cutoff number of mature oocytes needed to produce at least one or multiple top-quality embryos would have been different. We did not consider the pregnancy rate, so we could not analyze the cutoff number of mature oocytes needed to optimize the likelihood of establishing clinical pregnancy. A further large-scale study would be needed to determine the associations among mature oocyte number, the number of top-quality embryos, the clinical pregnancy rate, and the live birth rate. In conclusion, we demonstrated that the extreme cutoff numbers of mature oocytes were $\geq 9, \geq 9, \geq 10$, $\geq 10$, and $\geq 11$ for obtaining $\geq 1, \geq 2, \geq 3, \geq 4$, and $\geq 5$ top-quality embryos, respectively. We included normal responders only; however, our findings could help infertility clinicians to set target mature oocyte numbers in women undergoing stimulated IVF cycles.

\section{Conflict of interest}

Byung Chul Jee has been an editor of Journal of Clinical and Experimental Reproductive Medicine since 2018; however, he was not involved in the peer reviewer selection, evaluation, or decision process of this article. No other potential conflict of interest relevant to this article was reported.

\section{ORCID}

Yoo Jin Shim https://orcid.org/0000-0002-6895-3321

Yeon Hee Hong https://orcid.org/0000-0002-9709-4175

Seul Ki Kim https://orcid.org/0000-0002-1647-6711

Byung Chul Jee https://orcid.org/0000-0003-2289-6090

\section{Author contributions}

Conceptualization, Data curation, Formal analysis, Methodology, Project administration, Visualization, Writing-original draft, review \& editing: all authors.

\section{References}

1. Thurin A, Hausken J, Hillensjo T, Jablonowska B, Pinborg A, Strandell $A$, et al. Elective single-embryo transfer versus double-embryo transfer in in vitro fertilization. N Engl J Med 2004;351: 2392-402.

2. Baruffi RL, Mauri AL, Petersen CG, Nicoletti A, Pontes A, Oliveira 
JB, et al. Single-embryo transfer reduces clinical pregnancy rates and live births in fresh IVF and intracytoplasmic sperm injection (ICSI) cycles: a meta-analysis. Reprod Biol Endocrinol 2009;7:36.

3. Pandian Z, Templeton A, Serour G, Bhattacharya S. Number of embryos for transfer after IVF and ICSI: a Cochrane Review. Hum Reprod 2005;20:2681-7.

4. Fisch JD, Sher G, Adamowicz M, Keskintepe L. The graduated embryo score predicts the outcome of assisted reproductive technologies better than a single day 3 evaluation and achieves results associated with blastocyst transfer from day 3 embryo transfer. Fertil Steril 2003;80:1352-8.

5. Roberts SA, Fitzgerald CT, Brison DR. Modelling the impact of single embryo transfer in a national health service IVF programme. Hum Reprod 2009;24:122-31.

6. Taşdemir M, Tasdemir I, Kodama H, Fukuda J, Tanaka T. Two instead of three embryo transfer in in-vitro fertilization. Hum Reprod 1995;10:2155-8.

7. Terriou P, Sapin C, Giorgetti C, Hans E, Spach JL, Roulier R. Embryo score is a better predictor of pregnancy than the number of transferred embryos or female age. Fertil Steril 2001;75:525-31.

8. Volpes A, Sammartano F, Coffaro F, Mistretta V, Scaglione P, Allegra A. Number of good quality embryos on day 3 is predictive for both pregnancy and implantation rates in in vitro fertilization/ intracytoplasmic sperm injection cycles. Fertil Steril 2004;82: 1330-6.

9. Cai QF, Wan F, Huang R, Zhang HW. Factors predicting the cumulative outcome of IVF/ICSI treatment: a multivariable analysis of 2450 patients. Hum Reprod 2011;26:2532-40.

10. Lee TH, Chen CD, Tsai YY, Chang LJ, Ho HN, Yang YS. Embryo quality is more important for younger women whereas age is more important for older women with regard to in vitro fertilization outcome and multiple pregnancy. Fertil Steril 2006;86:64-9. 11. Sunkara SK, Rittenberg V, Raine-Fenning N, Bhattacharya S,
Zamora J, Coomarasamy A. Association between the number of eggs and live birth in IVF treatment: an analysis of 400135 treatment cycles. Hum Reprod 2011;26:1768-74.

12. Magnusson A, Wennerholm UB, Kallen K, Petzold M, ThurinKjellberg A, Bergh $C$. The association between the number of oocytes retrieved for IVF, perinatal outcome and obstetric complications. Hum Reprod 2018;33:1939-47.

13. Steward RG, Lan L, Shah AA, Yeh JS, Price TM, Goldfarb JM, et al. Oocyte number as a predictor for ovarian hyperstimulation syndrome and live birth: an analysis of 256,381 in vitro fertilization cycles. Fertil Steril 2014;101:967-73.

14. Kim JY, Kim JH, Jee BC, Lee JR, Suh CS, Kim SH. Can intracytoplasmic sperm injection prevent total fertilization failure and enhance embryo quality in patients with non-male factor infertility? Eur J Obstet Gynecol Reprod Biol 2014;178:188-91.

15. Bolton VN, Hawes SM, Taylor CT, Parsons JH. Development of spare human preimplantation embryos in vitro: an analysis of the correlations among gross morphology, cleavage rates, and development to the blastocyst. J In Vitro Fert Embryo Transf 1989;6:30-5.

16. Kim SK, Lee JR, Jee BC, Suh CS, Kim SH. What number of oocytes is appropriate for defining poor ovarian response? Yonsei Med J 2015;56:482-9.

17. Cai Q, Wan F, Huang K, Zhang H. Does the number of oocytes retrieved influence pregnancy after fresh embryo transfer? PLoS One 2013;8:e56189.

18. Hsu MI, Wang CW, Chen CH, Tzeng CR. Impact of the number of retrieved oocytes on pregnancy outcome in in vitro fertilization. Taiwan J Obstet Gynecol 2016;55:821-5.

19. Vermey BG, Chua SJ, Zafarmand MH, Wang R, Longobardi S, Cottell $E$, et al. Is there an association between oocyte number and embryo quality? A systematic review and meta-analysis. Reprod Biomed Online 2019;39:751-63. 